\title{
High-Throughput Printing via Microvascular Multinozzle Arrays
}

\author{
Christopher J. Hansen, Rajat Saksena, David B. Kolesky, John J. Vericella, Stephen J. Kranz, \\ Gregory P. Muldowney, Kenneth T. Christensen, and Jennifer A. Lewis*
}

High-throughput printing of functional materials in planar and three-dimensional motifs would enable numerous applications, including autonomic materials with embedded microvascularization, ${ }^{[1,2]} 3 \mathrm{D}$ scaffolds for tissue engineering ${ }^{[3]}$ and cell culture, ${ }^{[4,5]}$ lightweight structural composites, ${ }^{[6-9]}$ and printed electronics..$^{[10,11]}$ To date, direct laser ${ }^{[12,13]}$ and ink-writing methods ${ }^{[14,15]}$ have been primarily used to produce small components $\left(<1 \mathrm{~cm}^{3}\right.$ in volume), because lengthy fabrication times prohibit construction of larger structures. Yet, to transition 3D printing successfully from a prototyping to a true manufacturing platform, significant improvements in throughput are needed, particularly for applications requiring large form factors.

Recent efforts have focused on incorporating parallelization schemes into traditionally serial patterning techniques. Inkjet printing utilizes silicon-based parallel microfluidic printheads to eject multiple simultaneous microscale ink droplets. ${ }^{[16]}$ Massively parallel variants of dip-pen nanolithography, such as polymer pen lithography and hard-tip, soft-spring lithography, use polydimethylsiloxane (PDMS) or silicon multitip arrays to deposit low-viscosity functional inks on a substrate to yield 2D nanoscale patterns. ${ }^{[17,18]}$ Parallel electrospinning simultaneously deposits multiple nanofibers onto a substrate from addressable nozzles. ${ }^{[19,20]}$ Projection micro-stereolithography enables 3D fabrication via a digital micromirror dynamic mask, which selectively polymerizes voxels in parallel. ${ }^{[21]}$ However, each of these droplet-, filament-, or layer-based techniques is fundamentally limited to low viscosity inks, dilute precursor solutions, or photocurable organic resins; hence, they often require many deposition steps to achieve $3 \mathrm{D}$ structures. By contrast, direct-write assembly is a filamentary printing technique capable of patterning complex planar and $3 \mathrm{D}$ architectures ${ }^{[22]}$ with sub-micrometer to millimeter features from a diverse

Dr. C. J. Hansen, D. B. Kolesky, J. J. Vericella,

S. J. Kranz, Prof. J. A. Lewis

Department of Materials Science and Engineering

University of Illinois at Urbana-Champaign

Urbana, Illinois 61801, USA

E-mail: jalewis@illinois.edu

R. Saksena, Prof. K. T. Christensen

Department of Mechanical Science and Engineering

University of Illinois at Urbana-Champaign

Urbana, Illinois 61801, USA

Dr. G. P. Muldowney

The Dow Chemical Company

Electronic Materials, Newark, Delaware 19713, USA

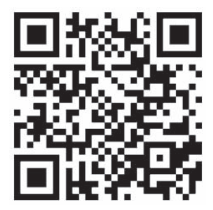

range of highly concentrated, viscoelastic inks. ${ }^{[23,24]}$ To date, however, this versatile printing method has been restricted to serial deposition from a single nozzle.

Here, we create multinozzle arrays composed of hierarchically branching, microvascular network(s) and demonstrate high-throughput printing of single and multiple materials over large areas $\left(\approx 1 \mathrm{~m}^{2}\right)$ (Figure 1). These printheads are designed to meet several criteria, including the ability to be produced in low-cost plastic, withstand the high pressures needed to print viscoelastic inks, generate uniform ink-flow rates across the nozzle array, enable continuous filamentary printing, and be coarrayed for multimaterial patterning. Our multinozzle design is readily scalable for large-area, rapid fabrication of planar and 3D functional architectures with microscale features.

The multinozzle printheads are designed to mimic fluid transport within the biological microvasculature, which consists of hierarchically branching channels. ${ }^{[25]}$ Each printhead distributes ink from a single (parent) microchannel into repeatedly bifurcated (child) branches to deposit ink simultaneously from multiple nozzles during printing. Each successive generation of bifurcating microchannels has dimensions that conform to Murray's law ${ }^{[26]}$ (i.e., $r_{0}^{3}=\sum_{i}^{n} r_{i}^{3}$, where $r_{0}$ is the radius of the parent channel and $r_{i}$ are the radii of the $n$ child channels). Murray's law can be extended to non-circular geometries by using the hydraulic diameter and a geometry-specific Poiseuille number. ${ }^{[27]}$ Although viscoelastic inks will exhibit a modified optimum, the conductance of symmetrically bifurcating networks results in uniform ink deposition.

For a bifurcating microvascular printhead composed of $k$ generations, the final generation will possess $2^{k}$ nozzles. To substantially increase the deposition rate, we produced a microvascular network consisting of six branching generations with a total of $64(k=6)$ nozzles. The microvascular channels have square cross-sections truncated in the sixth generation to yield nozzles that are $200 \mu \mathrm{m}$ on a side, which are suitable for printing both polymer and colloidal inks. To maintain minimum resistance for a branching network of fixed volume, the microchannels are self-similar (i.e., their channel lengths are proportional to their widths). ${ }^{[28]}$ The microchannel dimensions for each generation are provided in Figure $2 \mathrm{a}$.

To produce these multinozzle printheads, we first directly pattern the bifurcating network into a clear acrylic substrate using computer numeric controlled (CNC) micromachining (Figure 1a). The patterned substrate is then solvent-welded to a flat acrylic substrate (Figure 1b) to form a monolithic block that contains the embedded microvascular network (Figure 1c). Poly(methyl methacrylate) (PMMA) is chosen for the substrate material, due to its low cost, ease of machining, and ability

DOI: 10.1002/adma.201203321 


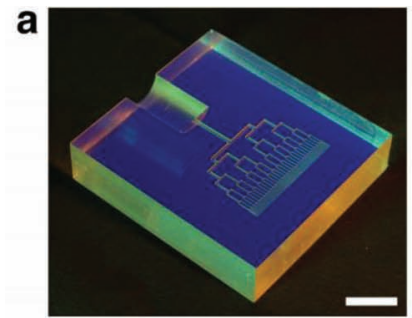

b
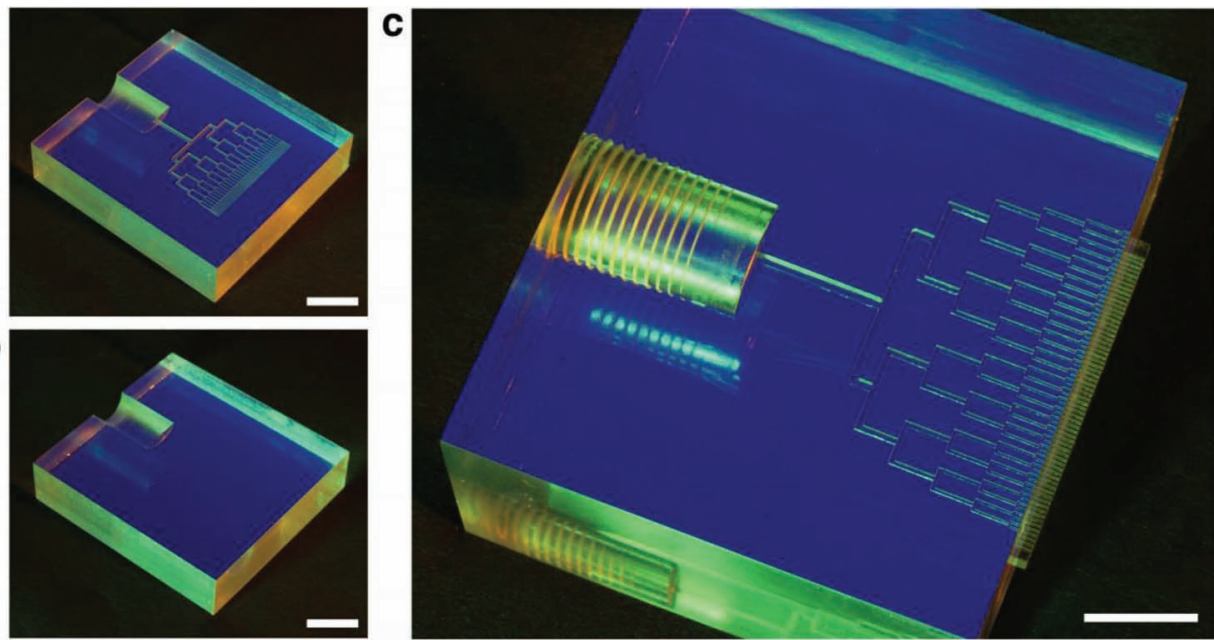

d

m

Figure 1. Microvascular multinozzle printhead. a-c) The hierarchical microvascular channel network is CNC-milled into an acrylic block (a), which is then solvent-welded to a nonpatterned acrylic block (b) to form an embedded microvascular structure within a monolithic block (c). The inlet is threaded and the bottom face of the device is milled and polished to expose the multinozzle array. The scale bars $=10 \mathrm{~mm}$. d) Optical micrograph of 64-nozzle array composed of square nozzles $(200 \mu \mathrm{m} \times 200 \mu \mathrm{m})$ with a $400 \mu \mathrm{m}$ center-to-center spacing. Scale bar $=2 \mathrm{~mm}$.

to be solvent-welded. We find that the welded blocks resist debonding under the highly pressurized (1-12 MPa) ink flow necessary to pattern viscoelastic inks. The nozzle dimensions (200 $\pm 5 \mu \mathrm{m}$ per side) vary by less than $2.5 \%$ (Figure $1 \mathrm{~d}$ ), which is within acceptable tolerances predicted by computer simulations described below.

Uniform ink flow is paramount to achieving high-fidelity, reproducible printed features. Resistance to flow within
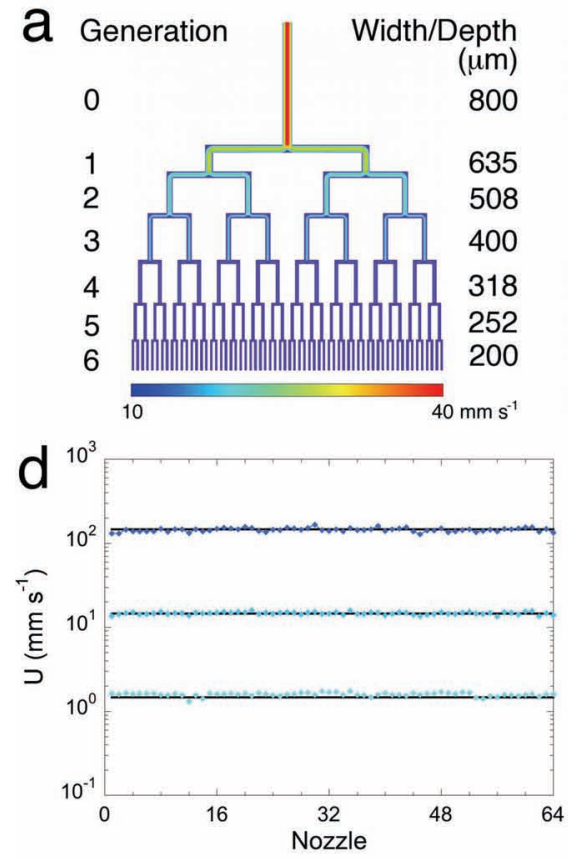
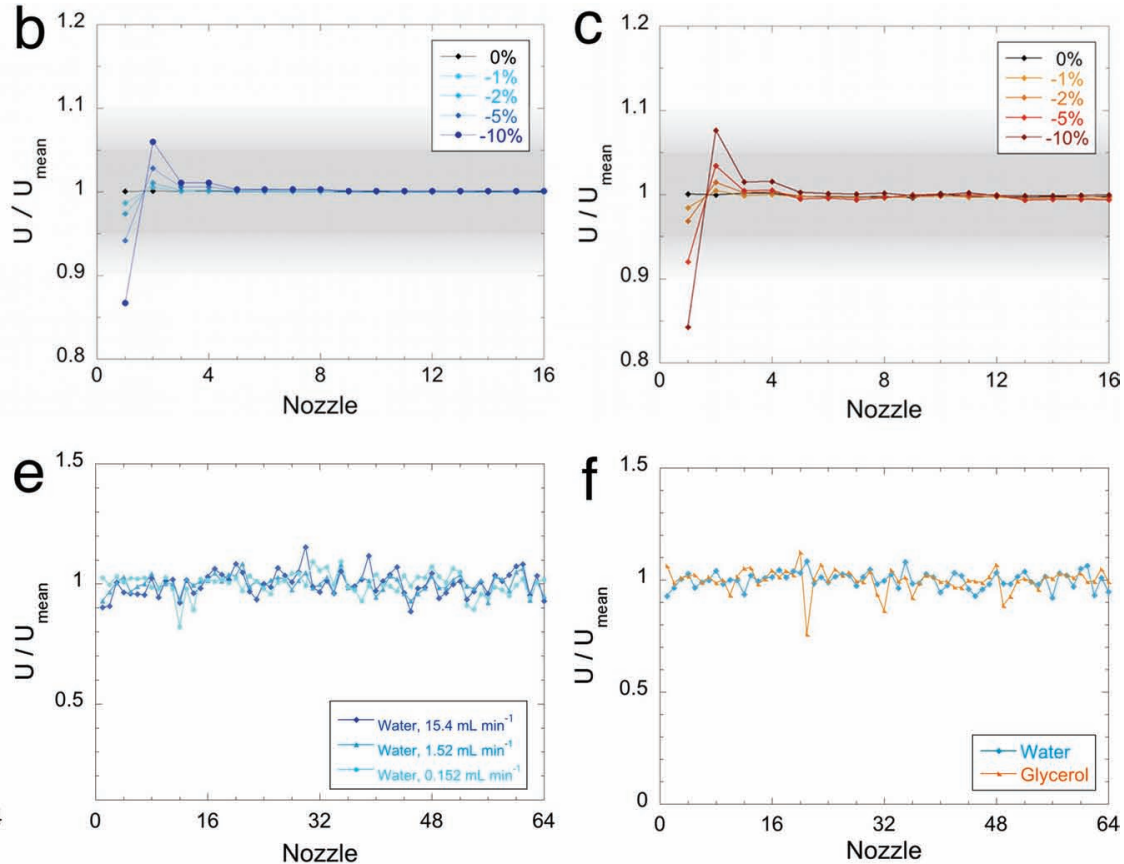

Figure 2. Fluid flow within a microvascular multinozzle printhead. a) Microvascular network dimensions and fluid-velocity map for 6-generation, 64-nozzle array. b,c) Modeling results for the average axial velocity $U$ of water (b) and wax ink (c) normalized by the mean outlet velocity $U_{\text {mean }}$ of $10 \mathrm{~mm} \mathrm{~s}^{-1}$ for all 64 nozzles. The dimensions of nozzle 1 are reduced by $0-10 \%$ from its original width of $200 \mu \mathrm{m}$. Note, the grey bands indicate acceptable variations in ink-flow velocity determined empirically from printing experiments. d,e) $\mu$-PIV measurements of the average axial velocity $U$ (d) and the normalized velocity $\left(U / U_{\text {mean }}\right.$ ) (e) for all of the nozzles for input flow rates spanning 2 orders of magnitude. The fluid is an aqueous suspension of $1 \mu \mathrm{m}$ fluorescent polystyrene tracer particles at a volume fraction, $\varphi$, of 0.00024 . f) Comparison of normalized velocity $U / U_{\text {mean }}$ for both water $(\varphi=$

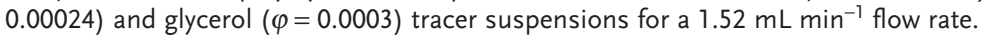


microchannels of width $w$ is inversely proportional to $w^{4}$; hence, even minor dimensional variations in their fabricated dimensions could give rise to non-uniformities in the ink flow among channels of a specific generation, which would become amplified in subsequent generations. To assess the precision required for printhead fabrication, we first perform computational fluid modeling. For the simplest case of a Newtonian fluid flowing in a perfectly symmetric bifurcating geometry, we find that a uniform flow velocity (i.e., standard deviation, $\sigma<0.001 U_{\text {mean }}$, where $U_{\text {mean }}$ is the mean of the average axial flow velocity $U$ across the channels) is achieved for steady-state laminar flow over a wide range of typical printing velocities $\left(1-100 \mathrm{~mm} \mathrm{~s}^{-1}\right)$ for both water and glycerol, which have respective viscosities $\mu$ of $1 \mathrm{cP}$ and $832 \mathrm{cP}$ at $22{ }^{\circ} \mathrm{C}$ (Supporting Information, Figure S1).

We systematically investigate the effects of asymmetric microvascular channel geometries, by selectively varying the width of a single channel leading into the first nozzle and measuring the resultant volumetric flow rate of that nozzle relative to the unmodified channels. We find that volumetric flow rate of water through the modified nozzle (nozzle 1) decreases monotonically, as its width is decreased by $1-10 \%$, while the widths of nozzles 2-64 remain fixed (Figure $2 \mathrm{~b}$ ). Fluid diverted from nozzle 1 is primarily routed to its neighboring nozzle (nozzle 2) in the same bifurcation generation. However, some additional flow is rerouted at the previous bifurcation generation $(k=5)$ and, hence, modest increases in fluid flow in nozzles 3 and 4 are also observed. For these conditions, there is a negligible change in flow rate for nozzles further removed from the altered nozzle. Analogous calculations carried out for glycerol show similar trends, though the flow deviations are slightly higher due to its increased viscosity.

We further investigate the precision needed to fabricate this printhead design by simulating the flow of a viscoelastic ink composed of microcrystalline wax, which conforms to a powerlaw fluid model. ${ }^{[2]}$ Specifically, we decrease the width of nozzle 1 by $1-10 \%$ (Figure 2c) and determine the resultant flow profiles, which indicate that dimensional changes of up to $5 \%$ (e.g., $10 \mu \mathrm{m}$ for a $200 \mu \mathrm{m}$ nozzle) can be tolerated before the mean velocity in the modified nozzle deviates by more than $10 \%$ from the base value. In related printing experiments, we find that continuous and reliable filamentary printing is achieved when the variation in ink-flow velocity among nozzles is less than $10 \%$. While these results are qualitatively similar to those for Newtonian fluids, the viscoelastic ink diverts $\approx 20 \%$ greater flow relative to water for a given nozzle-width constriction. Notably, for Newtonian fluids, the flow resistance of this microvascular network, which conforms to Murray's law, is roughly an order of magnitude lower than that calculated by the Hagen-Poiseuille equation for a printhead design composed of the same channel widths, but uniform channel depths of $200 \mu \mathrm{m}$. However, when microcrystalline wax is flowed at a rate of $10 \mathrm{~mm} \mathrm{~s}^{-1}$ through the 64-nozzle microvascular network, the calculated pressure drop $(430 \mathrm{MPa})$ is only a factor of two lower due to its strongly shear-thinning behavior.

Ink-flow uniformity within fabricated microvascular printheads is validated with microscopic particle image velocimetry ( $\mu$-PIV), which measures the fluid velocity fields within the microchannels. ${ }^{[2,30]}$ Specifically, the flow rates in the final generation of channels, which directly correspond to the nozzle flow rates, are interrogated by circulating dilute suspensions composed of fluorescent polystyrene tracer particles in either water or glycerol through a modified 6-generation bifurcating network that contains a fluid-collection reservoir (Supporting Information, Figure S2). The instantaneous fluid-velocity field is calculated by correlating fluorescent particle positions in pairs of time-delayed images. In direct ink writing, filamentary deposition occurs over a broad range of flow velocities, typically 1-100 $\mathrm{mm} \mathrm{s}^{-1}$ for a single nozzle. The input flow rates are chosen to mimic these printing conditions. The average nozzle velocity for each of the 64 nozzles are shown in Figure $2 \mathrm{~d}$ for target printing rates of 1,10 , and $100 \mathrm{~mm} \mathrm{~s}^{-1}$, which correspond to mean Reynolds numbers of $0.32<R e<28.8$, where $R e=U_{\text {mean }} L / v\left(U_{\text {mean }}=1.59\right.$ to $144.3 \mathrm{~mm} \mathrm{~s}^{-1}$ and characteristic length $L=200 \mu \mathrm{m})$. For each flow velocity, the standard deviation $\sigma$ as a fraction of the $U_{\text {mean }}\left(1.59,14.7\right.$, and $\left.144.3 \mathrm{~mm} \mathrm{~s}^{-1}\right)$ along the channel midplane is within $\pm 5 \%$. For a printing rate of $10 \mathrm{~mm} \mathrm{~s}^{-1}$, the midplane flow velocity $\left(U_{\text {mean }}=15.0 \mathrm{~mm} \mathrm{~s}^{-1}, \sigma=\right.$ $\left.0.052 U_{\text {mean }}\right)$ of glycerol is comparable to that of water $\left(U_{\text {mean }}=\right.$ $\left.14.7 \mathrm{~mm} \mathrm{~s}^{-1}, \sigma=0.036 U_{\text {mean }}\right)$. Most of the flow variation arises from variations in the syringe pump over the $\mu$-PIV capture period (i.e., $1-5 \%$ for $100-1 \mathrm{~mm} \mathrm{~s}^{-1}$, respectively). Any additional flow variation is attributed to minor imperfections in the fabricated microvascular geometry. Because $\mu$-PIV measurements are restricted to transparent fluids, velocity profiles for the microcrystalline wax cannot be obtained. However, direct imaging of microcrystalline wax flow through a microvascular printhead indicates that the ink velocity is nearly uniform across all 64 nozzles (Supporting Information, Movie S1).

High-throughput printing via microvascular multinozzle arrays is demonstrated using a 3-axis, motion-controlled stage capable of printing over a $1 \mathrm{~m}^{2}$ area at velocities up to $1 \mathrm{~m} \mathrm{~s}^{-1}$. The microcrystalline wax ink serves as a representative viscoelastic ink. Three printhead designs are initially fabricated and tested with $16(k=4), 32(k=5)$, and $64(k=6)$ nozzles, respectively. We note that the number of generations $k$ can be arbitrarily increased to produce printheads with $2^{k}$ nozzles. Each printhead contains an array of square nozzles evenly spaced across the printhead (25.6 $\mathrm{mm}$ width) with center-tocenter nozzle spacings of 1600 (Figure 3a), 800 (Figure 3b), and $400 \mu \mathrm{m}$ (Figure 3c), respectively. These designs result in volumetric fill factors of $12.5,25$, and $50 \%$, respectively. For the $50 \%$ volume fill factor, a single deposition pass simultaneously deposits 64 filaments (Figure $3 \mathrm{c}$ ) with little variation in filament height, $h$, and width, $w\left(\sigma_{\mathrm{h}}=0.025 h, \sigma_{\mathrm{w}}=0.030 w\right)$. Each multinozzle printhead enables ink filaments to be simultaneously deposited at printing velocities ranging from 1 to $100 \mathrm{~mm} \mathrm{~s}^{-1}$ with high fidelity and reproducibility.

To demonstrate large-scale patterning of a single material, we print wax ink onto a $1 \mathrm{~m}^{2}$ glass substrate using the 64-nozzle printhead (Figure 3d). 3D lattices are patterned over similarly large areas by rotating the printhead by $90^{\circ}$ between successive layers to create woodpile architectures (Figure 3e). An image of a representative $3 \mathrm{D}$ polymer lattice (4 layers, $0.61 \mathrm{~m} \times 0.61 \mathrm{~m}$ ) constructed by this approach is shown in Figure S3 in the Supporting Information. Alternate structures, including gradient or non-orthogonal designs, can be printed simply by altering the rotation angle of the printhead on-the-fly. 

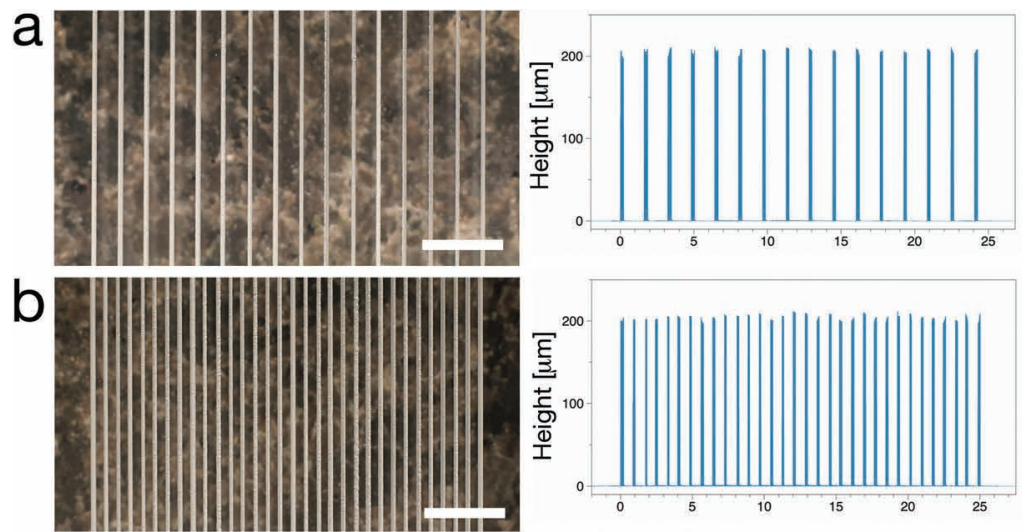

d
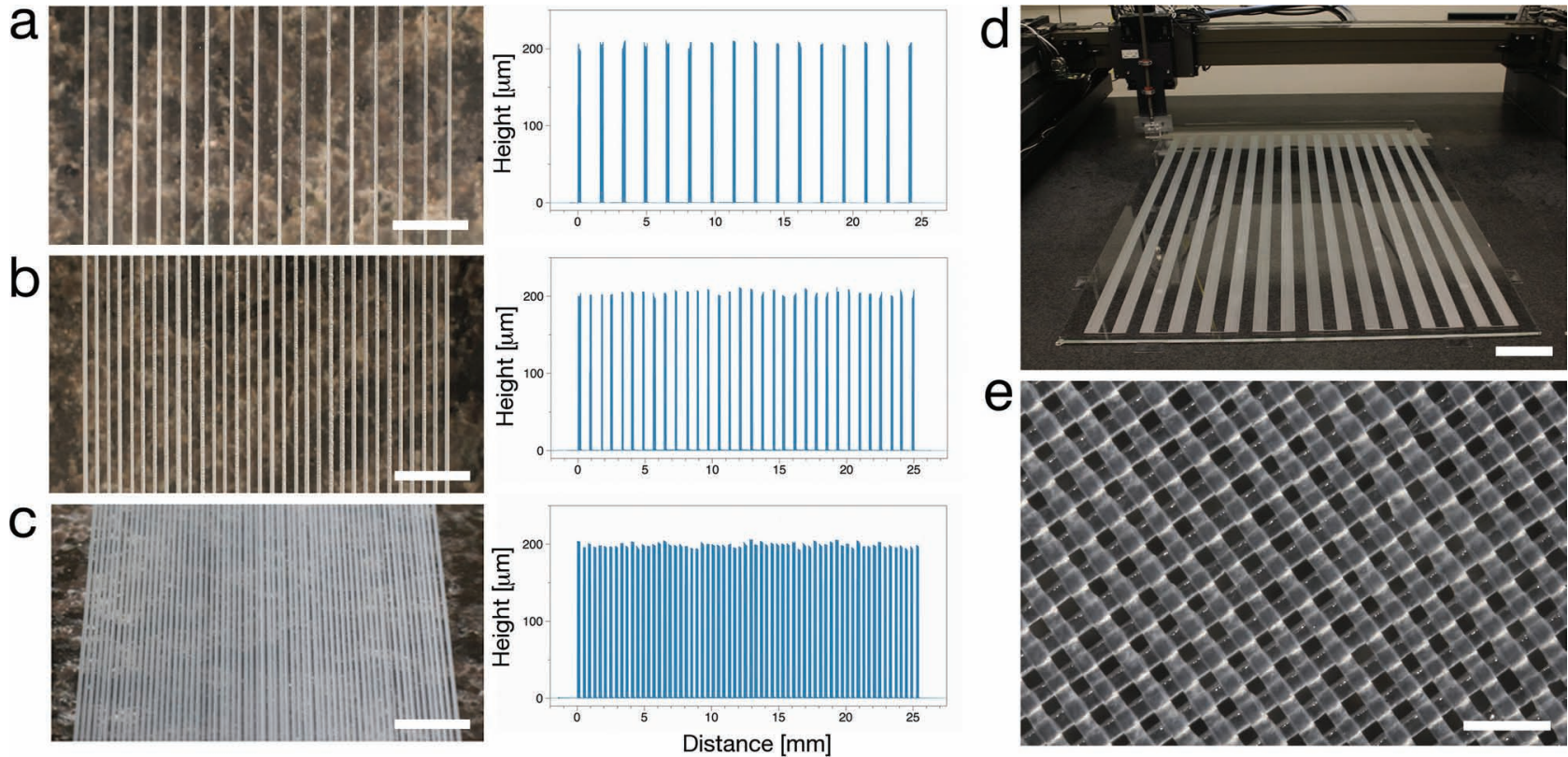

Figure 3. a-c) High-throughput printing of a single material using a microvascular multinozzle printhead. Optical image of ink filaments printed from 16 (a), 32 (b), and 64 (c) nozzle printheads in a single pass, with respective center-to-center spacings of 1600, 800, and $400 \mu \mathrm{m}$ (scale bars $=5 \mathrm{~mm}$ ). Corresponding height profiles of printed ink filaments measured by optical profilometry. d) Ink printing over large areas $(0.8 \mathrm{~m} \times 1.0 \mathrm{~m})$ onto a glass substrate (scale bar $=10 \mathrm{~cm}$ ). e) Optical image of a 2-layer lattice printed using a 64-nozzle printhead composed of $200 \mu \mathrm{m} \times 200 \mu \mathrm{m}$ nozzles with a $400 \mu \mathrm{m}$ center-to-center spacing (scale bar $=1 \mathrm{~mm}$ ).

To print multiple materials simultaneously, we conjoin two microvascular networks to form a dual multinozzle printhead (Figure 4a). Each multinozzle array is identical in its geometry and is fed by a separate ink reservoir. We begin by CNC-milling the microvascular networks into two separate PMMA substrates, and then solvent-welding their faces to opposite sides of a flat centerpiece. Their features are transversely offset with respect to the intended printing direction by a distance equal to half the nozzle center-to-center spacing (Supporting Information, Figure S4). During printing, the interstitial spaces present between ink filaments printed from the leading multinozzle array are infilled by printing the second ink from the trailing multinozzle array. The internal registration of this design eliminates alignment errors commonly observed in other multimaterial deposition schemes. As one example, we print a fully dense structure using two microcrystalline wax inks, one dyed blue and the other dyed yellow, which are co-flowed through this dual multinozzle printhead. The resulting multilayer structure is composed of a 3D bicontinuous architecture (Figure 4b). This scalable approach could be extended to enable simultaneous printing of three or more arbitrary materials composed of viscoelastic polymer, ceramic, or metallic inks developed previously. ${ }^{[1,4,6,7,11,24]}$

Another attribute of our dual multinozzle printhead design is the ability to pattern inks simultaneously with vastly different rheological properties. We demonstrate this by co-printing the viscoelastic wax ink with a Newtonian fluid composed of a photocurable epoxy resin (Supporting Information, Figure S4), which has a viscosity $\mu$ of $300 \mathrm{cP}$ between that of water and glycerol. The wax ink flows through the leading nozzle array, while the photocurable resin flows through the trailing nozzle array enabling that material to infiltrate the open space between printed wax filaments fully (Figure 4c). Despite extreme differences in their flow behavior, these materials can be successfully co-printed. This specific architecture is of interest for creating autonomic materials with embedded microvascular networks for self-healing,,$^{[1,31-33]}$ self-cooling, ${ }^{[2,34,35]}$ and pluripotent functionality. ${ }^{[36]}$ Dramatic reductions in printing time can be realized by implementing these multinozzle designs; for example, a $3 \mathrm{D}$ structure that requires a full day to print using a single nozzle can be fabricated in 22 min using a 64-nozzle printhead translated at the same printing speed.

In summary, we have developed microvascular multinozzle printheads for high-throughput printing of planar and multilayered architectures composed of single and multicomponent materials. Our nozzle design is inherently scalable, in that each additional bifurcation generation doubles the number of printing nozzles leading to further reductions in printing time. The minimum size of the printed filamentary features is governed by the nozzle dimensions, which can be as small as $\approx 10 \mu \mathrm{m}$ using CNC milling with even smaller dimensions achieved by lithographic techniques. Through the integration of microfluidic printhead designs with direct ink writing, we open new avenues for printing biomimetic architectures from functional materials, including self-healing and cooling polymer composites, lightweight foams, cellular constructs, and 3D scaffolds for tissue engineering.

\section{Experimental Section}

Materials: Microcrystalline wax ink composed of $40 \mathrm{w} / \mathrm{w} \%$ microcrystalline wax (SP-19, Strahl \& Pitsch, Inc.) and $60 \mathrm{w} / \mathrm{w} \%$ 


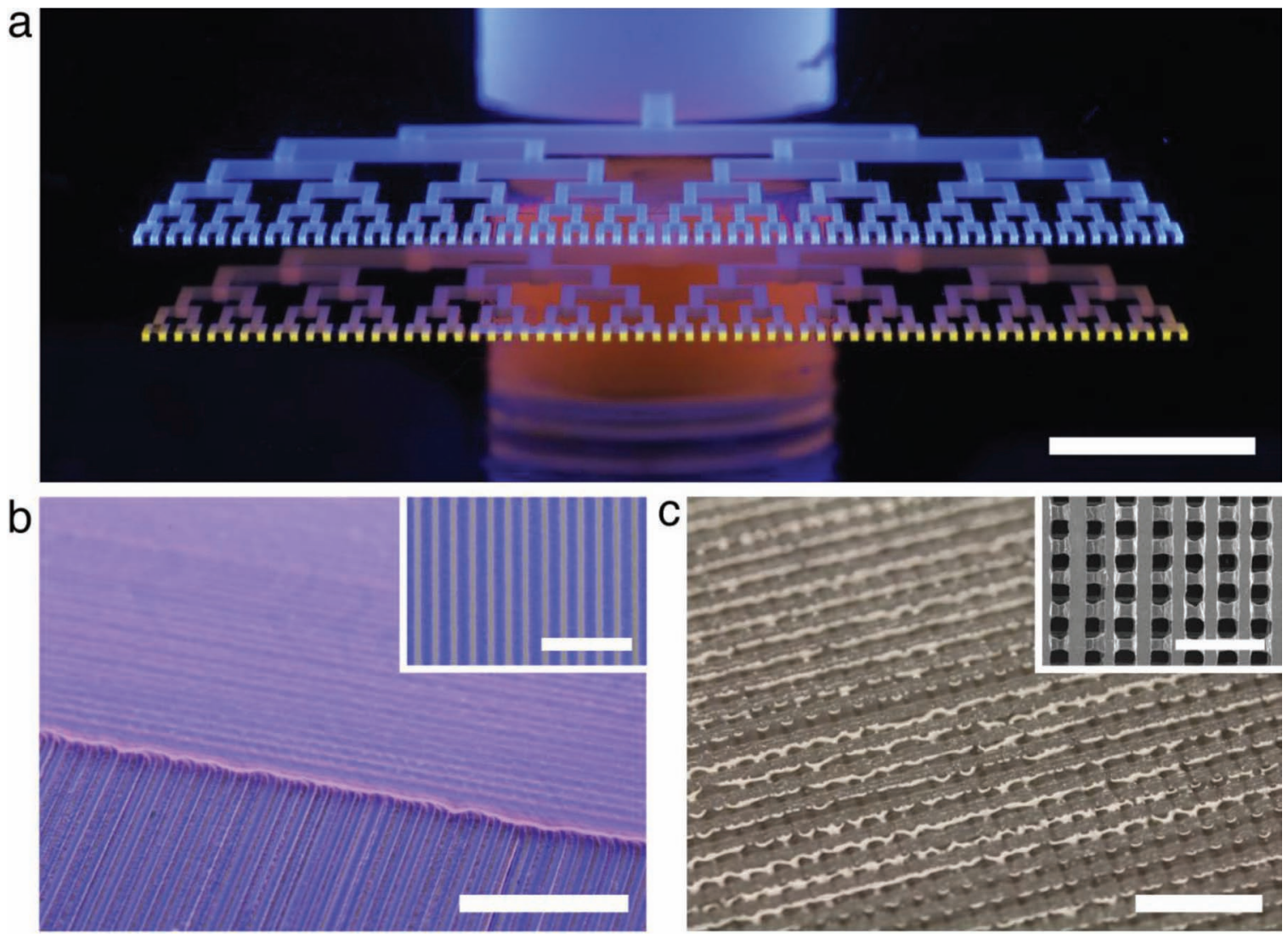

Figure 4. High-throughput printing of multiple materials using a dual microvascular multinozzle printhead. a) Optical image of the printhead filled with two inks fed by one ink inlet above (blue) and the other ink inlet below (yellow) (scale bar $=5 \mathrm{~mm}$ ). b) Optical image of printed multilayer structure composed of alternating wax filaments with blue and yellow fluorescent dyes (scale bar $=5 \mathrm{~mm}$ ), respectively. The inset shows a magnified view of a single layer region (scale bar $=2 \mathrm{~mm}$ ). c) Optical image of co-printed structure composed of wax ink filaments infilled with a photocurable epoxy resin $(\mathrm{scale}$ bar $=2 \mathrm{~mm})$. The inset shows a cross-section of a fully cured 10-layer structure with the fugitive wax ink removed (scale bar $=1 \mathrm{~mm})$.

petroleum jelly (Unilever, PLC.) was prepared by melting the components at $>100^{\circ} \mathrm{C}$ and subsequently mixing. The molten ink was filtered through a $25 \mathrm{~mm}$ diameter, $2 \mu \mathrm{m}$ glass-fiber filter (Millex AP 20 prefilter, Millipore Corp.) to remove debris and particulates. The mixture was poured into a heated pump reservoir (500D, Teledyne, Inc.) and was slowly cooled to room temperature overnight.

For the $\mu$-PIV experiments, dilute suspensions were prepared by suspending polystyrene tracer particles $(1 \mu \mathrm{m}$ diameter, $\varphi=0.00024-$ 0.00030 ) dyed with nile red (F-8819, Invitrogen) in either deionized water or glycerol. The suspensions were ultrasonicated $(20 \mathrm{~min})$ prior to loading into $60 \mathrm{~mL}$ syringes (BD Syringe).

Multinozzle Fabrication: Poly (methyl methacrylate) (PMMA) polymer blocks $(50 \mathrm{~mm} \times 50 \mathrm{~mm} \times 25 \mathrm{~mm}$ ) were mounted on a CNC-mill $(8540$, Sherline Products Inc.). Features $508 \mu \mathrm{m}$ or smaller were milled with a $200 \mu \mathrm{m}$ square-end end mill (Ultra-Tool International), while features $635 \mu \mathrm{m}$ or larger were milled with a $635 \mu \mathrm{m}$ end mill. The channel depths were measured using an optical profilometer (LT-9010M, Keyence) and the piece was polished on a polishing wheel (EcoMet, Buehler Inc.) until the channel depths were within $5 \mu \mathrm{m}$ of the intended depth and each channel width was within $\pm 2 \mu \mathrm{m}$ of the intended dimension for all of the microchannels. The reservoir input(s) were machined and threaded and the microchannels are then filled with a fugitive wax (Purester 24, Strahl \& Pitsch) to prevent channel deformation during solvent-welding. A non-patterned PMMA block was set on a parafilm release layer and acetonitrile was pipetted onto the PMMA surface. The milled PMMA block was set in contact with the flat piece and immediately compressed in an isostatic press (Model C, Carver Inc.) at $\approx 35 \mathrm{MPa}$ for a minimum of $15 \mathrm{~min}$. The nozzles were exposed through a combination of milling and polishing and each output length was verified to be equal within $\pm 10 \mu \mathrm{m}$ using an optical microscope (DMI 6000B, Leica Microsystems). Finally, the part face surrounding the nozzles was recessed by $\approx 2 \mathrm{~mm}$ relative to the outputs to decrease the possible interactions between the printhead and the printed structures.

Dual multinozzle printheads were produced by creating a welded piece as described above and flipping the piece. A second part containing a milled network was then aligned to the first piece using a jig and solvent-welded under pressure. Proper network alignment was verified using optical microscopy.

Fluid Modeling: Flow through the channel network was modeled and solved using the ANSYS FLUENT v.13.0 software package. The 3D microvascular geometry was developed and meshed as a structured grid with hexahedral volume elements in GAMBIT (ANSYS Inc.). The FLUENT finite volume-based solver was used to solve the Navier-Stokes equations through the meshed domain. Constant inlet mass flow, constant outlet pressure, and no-slip boundary conditions were applied. A segregated solver was used to solve the field equations for a steadystate laminar flow. The unsteady formulation was first order implicit. The pressure was discretized using the pressure staggering option (PRESTO) scheme. The semi-implicit pressure linked equations (SIMPLE) algorithm was employed to ensure pressure-velocity coupling. Convective terms in the momentum-conservation equations were discretized using the firstorder upwind scheme.

$\mu$-PIV Experiments: All of the $\mu$-PIV flow quantifications were performed on a single microvascular network that was modified to 
include a collection reservoir to capture the flow output (see Supporting Information, Figure S3). The surface was encapsulated with an optically transparent pressure-sensitive adhesive layer ( $3 \mathrm{M}$ Inc.). The syringe pump (PHD2000, Harvard Apparatus) flow rate was calibrated by flowing water through the network to a humidity-controlled balance chamber (AG285, Mettler Toledo) and collecting data in LabView (v.2009, National Instruments).

$\mu$-PIV measurements were recorded for a constant suspension flow rate after an initial start-up transient. A double-pulsed Nd:YAG laser (Solo PIV, New Wave Research) was directed through a 10x objective (numerical aperture (N.A.) $=0.30$, working distance $=10.0 \mathrm{~mm}$, depth of field $=14.72 \mu \mathrm{m}$ ) on an upright microscope (Olympus BX60) and was captured by a frame-straddle charge-coupled-device (CCD) camera (11 MP PowerView Plus, TSI) with a synchronizer (Model 610034, TSI Inc.). The laser pulses ( $\lambda=532 \mathrm{~nm}, \approx 10 \mathrm{~mJ}$ per pulse, $5 \mathrm{~ns}$ pulse width) were guided through an epifluorescent filter cube with a dichroic beam splitter followed by the objective lens to volume illuminate the field of view. The fluorescent particles $\left(\lambda_{\text {peak }}=575 \mathrm{~nm}\right)$ were imaged at the midplane of each channel. The time delay for each flow rate measurement was chosen to yield a mean particle displacement of 8-12 pixels, and 25 image pairs were captured for each measurement.

The image pairs acquired were analyzed using a recursive, two-frame cross-correlation algorithm with $50 \%$ overlap between the adjacent firstinterrogation windows. A first-pass interrogation allowed the algorithm to employ the offset of the second-interrogation window in the secondinterrogation pass more intelligently. Both passes used a spot size of $32 \times 32$ pixels, which yielded a vector grid spacing of $\Delta x=\Delta y=$ $26 \mu \mathrm{m}$. Since the flow was steady, an ensemble correlation algorithm was employed, yielding a single, ensemble-averaged vector field for each measurement. ${ }^{[37]}$ Each vector field was then validated using local median and mean magnitude differences to remove erroneous vectors and fill in small holes with interpolated vectors where at least $50 \%$ of neighbors were present. Each vector field was then low-pass filtered with a narrow Gaussian filter to reduce noise associated with frequencies larger than the sampling frequency of interrogation.

High-Throughput Printing: Ink was flowed from a reservoir (260D Syringe Pump, Teledyne Isco) into a multinozzle printhead via a high-pressure hose (US Hose Corporation). The printhead was mounted to the print stage (ABG10000, Aerotech Inc.) so that it was parallel to the substrate. The height profile of a large glass substrate was obtained using an optical profilometer (LT-9010M, Keyence), such that, during printing, the height between the multinozzle printhead and the substrate was controlled to within $200 \mu \mathrm{m} \pm 5 \mu \mathrm{m}$ over the $1 \mathrm{~m}^{2}$ area. The fly-height corresponded to the characteristic dimensions of the individual nozzles within the array. Viscoelastic ink material was deposited at velocities and applied pressures of $1-50 \mathrm{~mm} \mathrm{~s}^{-1}$ and 2-6 MPa, respectively. For multiple-layer structures, the printhead was rotated by $90^{\circ}$ between each layer.

Co-printing of a photocurable epoxy resin with the microcrystalline wax ink was performed by filling a $60 \mathrm{~mL}$ syringe with NOA-61 (Norland Products Inc.). The syringe was loaded onto a syringe pump (PHD2000, Harvard Apparatus) and fitted with a luer connection to $6 \mathrm{~mm}$ inner diameter PVC tubing to transport fluid to the second inlet on the dual microvascular multinozzle printhead. During printing, the syringe pump was set to flow rates sufficient to fill the interstitial spaces between printed wax filaments.

\section{Supporting Information}

Supporting Information is available from the Wiley Online Library or from the author.

\section{Acknowledgements}

The authors gratefully acknowledge support provided by the Dow Chemical Company (Award\# 2006-06842) and the National Science
Foundation (Grant\# DMR-0652424). C.J.H. is supported in part by a National Science Foundation Graduate Student Fellowship.

Received: August 11, 2012

Published online: October 26, 2012

[1] C. J. Hansen, W. Wu, K. S. Toohey, N. R. Sottos, S. R. White, J. A. Lewis, Adv. Mater. 2009, 21, 4143.

[2] B. D. Kozola, L. A. Shipton, V. K. Natrajan, K. T. Christensen, S. R. White, J. Intell. Mater. Syst. Struct. 2010, 21, 1147.

[3] L. Sun, S. T. Parker, D. Syoji, X. Wang, J. A. Lewis, D. L. Kaplan, Adv. Healthcare Mater. 2012, DOI: 10.1002/adhm. 201200057.

[4] J. N. Hanson Shepherd, S. T. Parker, R. F. Shepherd, M. U. Gillette, J. A. Lewis, R. G. Nuzzo, Adv. Funct. Mater. 2011, 21, 47.

[5] R. A. B. III, R. F. Shepherd, J. N. Hanson, R. G. Nuzzo, P. Wiltzius, J. A. Lewis, Adv. Mater. 2009, 21, 2407.

[6] B. Y. Ahn, D. Shoji, C. J. Hansen, E. Hong, D. C. Dunand, J. A. Lewis, Adv. Mater. 2010, 22, 2251.

[7] E. Hong, B. Y. Ahn, D. Shoji, J. A. Lewis, D. C. Dunand, Adv. Eng. Mater. 2011, 13, 1122.

[8] T. A. Schaedler, A. J. Jacobsen, A. Torrents, A. E. Sorensen, J. Lian, J. R. Greer, L. Valdevit, W. B. Carter, Science 2011, 334, 962.

[9] G. E. Fair, M. Y. He, R. M. McMeeking, F. F. Lange, J. Am. Ceram. Soc. 2005, 88, 1879.

[10] B. Y. Ahn, E. B. Duoss, M. J. Motala, X. Guo, S.-I. Park, Y. Xiong, J. Yoon, R. G. Nuzzo, J. A. Rogers, J. A. Lewis, Science 2009, 323 1590.

[11] B. Y. Ahn, D. J. Lorang, J. A. Lewis, Nanoscale 2011, 3 , 2700.

[12] D. Lim, Y. Kamotani, B. Cho, J. Mazumder, S. Takayama, Lab Chip 2003, 3, 318.

[13] D. H. Kam, J. Mazumder, J. Laser Appl. 2008, 20, 185.

[14] H. Sirringhaus, T. Kawase, R. H. Friend, T. Shimoda, M. Inbasekaran, W. Wu, E. P. Woo, Science 2000, 290, 2123.

[15] D. Tobjörk, R. Österbacka, Adv. Mater. 2011, 23, 1935.

[16] P. Calvert, Chem. Mater. 2001, 13, 3299.

[17] W. Shim, A. B. Braunschweig, X. Liao, J. Chai, J. K. Lim, G. Zheng, C. A. Mirkin, Nature 2011, 469, 516.

[18] F. Huo, Z. Zheng, G. Zheng, L. R. Giam, H. Zhang, C. A. Mirkin, Science 2008, 321, 1658.

[19] M. R. Ladd, S. J. Lee, J. D. Stitzel, A. Atala, J. J. Yoo, Biomaterials 2011, 32, 1549

[20] N. Q. Zhan, Y. X. Li, C. Q. Zhang, Y. Song, H. G. Wang, L. Sun, Q. B. Yang, X. Hong, J. Colloid Interface Sci. 2010, $345,491$.

[21] C. Sun, N. Fang, D. M. Wu, X. Zhang, Sensors Actuators A: Phys. 2005, 121, 113

[22] G. M. Gratson, M. Xu, J. A. Lewis, Nature 2004, 428, 386.

[23] J. A. Lewis, J. Am. Ceram. Soc. 2000, 83, 2341

[24] J. A. Lewis, Adv. Funct. Mater. 2006, 16, 2193.

[25] A. R. Pries, T. W. Secomb, in Handbook of Physiology: Microcirculation, (Eds.: R. F. Tuma, W. N. Duran, K. Ley), Academic Press, San Diego, CA USA 2008.

[26] C. D. Murray, Proc. Natl. Acad. Sci. USA 1926, 12, 207.

[27] D. R. Emerson, K. Cieślicki, X. Gu, R. W. Barber, Lab Chip 2006, 6, 447.

[28] T. F. Sherman, J. Gen. Physiol. 1981, 78, 431.

[29] D. Therriault, S. R. White, J. A. Lewis, Appl. Rheol. 2007, 17, 10112

[30] V. K. Natrajan, K. T. Christensen, Exp. Fluids 2007, 43, 1.

[31] C. J. Hansen, S. R. White, N. R. Sottos, J. A. Lewis, Adv. Funct. Mater. $2011,21,4320$. 
[32] A. R. Hamilton, N. R. Sottos, S. R. White, Adv. Mater. 2010, 22, 5159.

[33] S. C. Olugebefola, A. M. Aragon, C. J. Hansen, A. R. Hamilton, B. D. Kozola, W. Wu, P. H. Geubelle, J. A. Lewis, N. R. Sottos, S. R. White, J. Compos. Mater. 2010, 44, 2587.

[34] R. B. Oueslati, D. Therriault, S. Martel, IEEE Trans. Compon. Packag. Technol. 2008, 31, 869.
[35] B. Dang, M. S. Bakir, D. C. Sekar, C. R. King, J. D. Meindl, IEEE Trans. Adv. Packag. 2010, 33, 79.

[36] K. Saha, Y. Mei, C. M. Reisterer, N. K. Pyzocha, J. Yang, J. Muffat, M. C. Davies, M. R. Alexander, R. Langer, D. G. Anderson, R. Jaenish, Proc. Natl. Acad. Sci. USA 2011, 108, 18714.

[37] C. D. Meinhart, S. T. Wereley, J. G. Santiago, J. Fluids Eng. 2000, 122, 285. 